\title{
Analysis of Distributed Wireless Sensor Systems with a Switched Quantizer
}

\author{
Hui Sun (D), ${ }^{1}$ Xianyu Wang, ${ }^{2}$ Kaixin Yang, ${ }^{2}$ and Tongrui Peng $\mathbb{D}^{3}$ \\ ${ }^{1}$ College of Electronic Information and Automation, Civil Aviation University of China, South Campus, Tianjin 300300, China \\ ${ }^{2}$ Tianjin College, University of Science and Technology Beijing, Tianjin 301830, China \\ ${ }^{3}$ College of Science, Shanghai Institute of Technology, Shanghai 200235, China
}

Correspondence should be addressed to Tongrui Peng; tongrui2.peng@sit.edu.cn

Received 17 November 2020; Accepted 17 July 2021; Published 29 July 2021

Academic Editor: Jing $\mathrm{Na}$

Copyright ( $\odot 2021$ Hui Sun et al. This is an open access article distributed under the Creative Commons Attribution License, which permits unrestricted use, distribution, and reproduction in any medium, provided the original work is properly cited.

In this article, a switched quantizer is proposed to solve the bandwidth limitation application problem for distributed wireless sensor networks (WSNs). The proposed estimator based on switched quantitative event-triggered Kalman consensus filtering (KCF) algorithm is used to monitor the aircraft cabin environmental parameters when suffering packet loss and path loss issues during the communication process for WSN. The quantization error of the novel switched quantizer structure is bounded, and the corresponding stability theory for the quantitative estimation approach is proved. Compared with other methods, the simulation results for the introduced method verify that the environmental parameters can be estimated accurately and timely and reduce the burden of network communication bandwidth.

\section{Introduction}

Due to the rapid development of the civil aviation industry, the safe and comfortable cabin environment has become one of the most important foci for aircraft designers and operators. Passengers and crew members have also imposed higher requirements on the cabin environment. Once mechanical failures, pipe ruptures, or seal failures occur in the narrow and closed cabins and cargos, the cabin environment probably is contaminated, which can cause passengers and crew members dizziness, headaches, ear disease, dry eye syndrome, throat pains, and other adverse events, even severe neurological disorders $[1,2]$. This will seriously threaten flight safety. The higher air recirculation rate is a trend for the design of future aircraft for guaranteeing air quality in the cabin [3]. Currently, aircraft cabins install fire monitoring systems. However, false alarms often occur in reality. Therefore, it is necessary to develop a method to estimate environmental parameters and monitor sudden environmental issues accurately in cabins. With the rapid development of wireless sensing technology, the design of aircraft will move from the current fly-by-wire system to the fly-by-wireless direction [4]. Wireless sensor network (WSN) combines sensor technology, wireless communication, information fusion, and other technologies, a multidisciplinary technology. At present, WSN has shown great application value and broad business vision in applications such as environmental testings, military, health, agriculture, and other areas. As an emerging application of information technology, WSN realizes the function of collaborative sensing and processing the physical information of the detected objects through wireless transmission and information interaction and provides direct, effective, and real information support for real-time monitoring and fast processing detection time $[5,6]$. Also, it becomes possible that some specific WSNs such as short-range WSN can be employed in monitoring narrow and complex spaces, such as cabin and cargo environments which are the trend for the future aircraft industry. Then, estimating environment parameters accurately and quickly is the first prior issue for the correct alarm decision to monitor inside environments.

Simultaneously, many researchers focus on distributed state estimation algorithms over distributed WSNs. Furthermore, it is well known that the Kalman filter can help 
filter noises applied to systems. Therefore, the above researches are always combined with Kalman filters. For example, Yang et al. apply the sensor network for distributed estimation as the research background, review the Kalman consensus filtering problem, systematically introduce various Kalman uniform filtering related algorithms, and summarize the research status in this field in detail [7]. Olfati-Saber proposes a scalable distributed Kalman consensus algorithm [8]. The work in [9] further studies the optimality of the discrete form of the algorithm and also establishes stability analysis. The study in [10] proposes independent consensus matrices with each neighbor's information. Most of the above research works are carried out under ideal communication preconditions. For example, the network bandwidth is infinite and the communication information is the ideal value.

However, these assumptions are difficult to implement in practical applications. In the real world, bandwidth is limited. A feasible way to solve the above problem is to insert quantizers in the systems, such as from sensor to controller or from the controller to the actuator. The quantizer here can be summarized as a class of quantizers that satisfy regional bounded conditions, such as logarithmic quantizers, and uniform quantizers [11]. The introduction of quantizers will inevitably introduce quantization errors, which may degrade system performance and even cause system instability. Therefore, it is an important direction in the current research of WSNs to study the statistical characteristics and error types introduced by quantization and minimize the quantization errors. In 1956, Kalman et al. proposed to add a quantizer to the control system. Later, in the study of some related quantization algorithms, the quantization error was generally treated as an augmented noise. Ribeiro et al. propose a quantized Kalman filter considering the communication constraints in the network, which made only one sensor activated in each sampling period of the distributed filtering $[12,13]$. In $[14,15]$, an adaptive quantization-based input quantization control scheme is proposed for a class of linear systems. The work in [16] studies the input quantization control problem for a class of nonlinear systems. In [17], the Lyapunov method is used to study the quantization control problem of a class of nonlinear systems with time delays. The study in [18] describes the latest developments in quantitative control of linear systems. In [19], the quantization control problem for input and output quantization is considered. The work in [20] proposes a Kalman filtering algorithm based on event-triggered consensus for cabin pollutants monitoring. However, it does not consider the network bandwidth pressure of the system. The study in [21] introduces a hybrid trigger scheme and quantization and studies the quantization state estimation problem of neural networks with network attacks and hybrid trigger communication schemes to reduce the pressure of network transmission and save network resources. In [22], several operational modes are designed for quantizers and estimators and a nonfragile $H_{\infty}$ estimation problem for a class of complex networks with switched topologies and quantization effects is studied. The work in [23] focuses on the linear least mean square estimation of network discrete time-varying linear systems affected by data quantization and communication constraints. The study in [24] proposes a new descriptor sliding mode observer method to solve the problem that signal quantization will reduce the estimation performance. In [25], the quantization state estimation suitable for general vector measurement is derived based on Bayesian theory. The work in [26] studies a recursive filtering algorithm to deal with state estimation problems in power systems with quantized nonlinear measurements. In [27], a linear distributed Luenberger observer is proposed to ensure that the estimation error of distributed state estimation is bounded for quantized multiagent data in the case of a limited communication data rate. In [28], quantization of data with the Laplacian distribution is considered in networks. The work in [29] deals with the event-triggered fuzzy filtering issue by considering data quantization in-vehicle sideslip angle estimation.

Therefore, inspired by the above literature, this paper designs a switched quantizer for the WSN-based pollutant concentration estimation algorithm to solve the communication bandwidth limitation. This is consecutive to [20]. The proposed event-trigger based algorithm with quantizers can not only help accurately estimate the cabin environment parameters in time but can also save communication bandwidth.

According to the motivation mentioned above, an analysis method is addressed via a switched quantizer. In summary, two contributions of this paper are given as follows:

(1) An estimation algorithm with consideration of event-trigger of distributed wireless sensor networks is proposed. A switched quantizer is used to reduce the occupancy of bandwidth for transmitting information by quantizing the data from the observers. The proposed approach can save the communication bandwidth for path loss and random packet loss events.

(2) A series of stability theorems are deduced and provided for the proposed switched quantizer-based estimation algorithm.

The following contents of this paper are arranged as follows. Section 2 introduces the preliminary knowledge and system model including the monitoring network and the integrated sensing error model. Section 3 proposes the event-triggered switched quantizer-based Kalman consensus filtering algorithm in the event of interferences. Furthermore, stability analyses of the algorithm and the sufficient conditions for bounded mean square exponential estimation error of the algorithm are given. Section 4 demonstrates simulation results that verify the effectiveness of the algorithm proposed in this paper. Conclusions are summarized in the last section.

\section{Preliminaries}

2.1. Integrated Sensor Structure. In aircraft cabins, there are various kinds of pollutants generated by sudden environmental events. The measurement results are always sensitive 
to interferences by using one single sensor. Therefore, it is necessary to construct WSN with integrated nodes to estimate concentrations in cabins. The most mature field for the application of integrated sensing structures is the integrated navigation system of aircraft. The relevant introduction can be found in [30].

This paper will select an output correction structure as the node structure and use KCF. The comparison among several integrated measurement structures is proposed in [31]. Based on this integrated sensor measurement structure, the study in [20] proposes integrated sensor nodes to construct a distributed integrated sensor monitoring network and presents an event-triggered Kalman consensus filter algorithm (ET-KCF). A monitoring network is shown in Figure 1 where the dotted line is the neighbor node of node $i$. The measurement result of the primary sensor is $Z_{P i}$, and $Z_{S i}$ is that of the secondary sensor. $Z_{i}$ is the difference between $Z_{P i}$ and $Z_{S i}$. It is fused with estimated information of the neighbors based on the ET-KCF method. Then the measured value coming from the primary sensor is corrected by using the error estimate to get the optimal estimate value of concentration, $Z_{\mathrm{O} i}$.

The topology diagram of the wireless sensor network is defined as $G=(V, E, A)$, where $V=\left\{v_{1}, v_{2}, \ldots, v_{n}\right\}$ is the set of sensor nodes and $E=V \times V$ is the set of edges between nodes. The set of neighbors of the sensor $i$ is denoted by $N_{i}=\left\{v_{j} \in V \mid\left(v_{i}, v_{j}\right) \in E\right\}$, while the number of its neighbors is called the degree of the sensor node and denoted by $d_{i}=\left|N_{i}\right|$. The degree matrix $D$ of the topological graph $G$ is defined as a diagonal matrix of diagonal elements of each sensor node degree, and denoted by $D=\operatorname{diag}\left\{d_{1}, \ldots, d_{N}\right\}$, $A=\left[a_{i j}\right]$ is an adjacency matrix representing the communication relationship among sensors. When sensor $i$ and the sensor $j$ communicate with each other, $a_{i j}$ takes a value of 1 , otherwise 0 . The Laplacian matrix of graph $G$ is represented as $L=D-A$. If the matrix $L$ has a nonzero eigenvalue, then the undirected topological graph $G$ is connected [32].

Referring to [31], this paper discusses a general environment parameter monitoring algorithm model without considering the specific concentration parameter monitoring dynamic model.

As shown in Figure 1, the ET-KCF is performed on the measuring errors between the primary sensor and the secondary sensor. Suppose the primary and secondary sensor dynamic equations of the integrated sensors are shown in equations (1) and (2).

The primary sensor dynamic equation is shown in the following equation:

$$
\left\{\begin{array}{l}
x_{p}^{+}=A_{p} x_{p}+B_{p} w_{p}, \\
z_{p i}=H_{p i} x_{p}+F_{p i} v_{p i},
\end{array}\right.
$$

and the secondary sensor dynamic equation is shown in the following equation:

$$
\left\{\begin{array}{l}
x_{s}^{+}=A_{s} x_{s}+B_{s} w_{s} \\
z_{s i}=H_{s i} x_{s}+F_{s i} v_{s i}
\end{array}\right.
$$

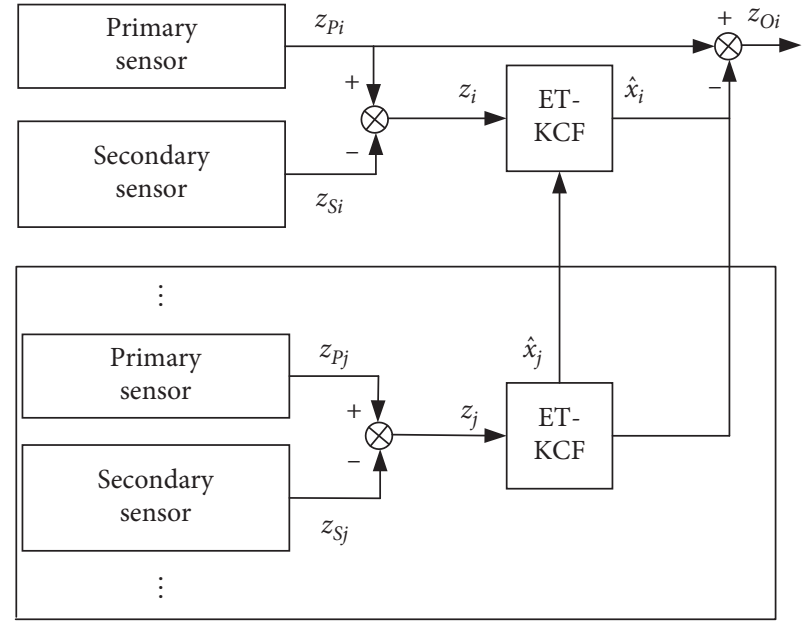

FIgURE 1: Schematic diagram of a distributed integrated sensing monitoring network [19].

where superscript + means the updated value of variables. $x_{p} \in R^{m p}$ and $x_{s} \in R^{m s}$ are state vectors of the primary and secondary sensors. $z_{p i} \in R^{p}$ and $z_{s i} \in R^{s}$ are output vectors of the primary and secondary sensors. $\left(A_{p}, B_{p}, H_{p i}, F_{p i}\right)$ and $\left(A_{s}, B_{s}, H_{s i}, F_{s i}\right)$ are system matrices with appropriate dimensions. $w_{p}, v_{p i}, w_{s}$, and $v_{s i}$ are independent white Gaussian noises and their covariance matrices satisfy

$$
\begin{aligned}
& E\left[w(k) w(l)^{T}\right]=Q(k) \delta_{k l}, \\
& E\left[v_{i}(k) v_{j}(l)^{T}\right]=R_{i j}(k) \delta_{k l},
\end{aligned}
$$

where $\delta_{k l}$ is an impulse response function. When $k=l, \delta_{k l}$ equals 1 , otherwise $0 . Q$ and $R_{i j}$ are the corresponding noise covariance matrices. When $i=j, R_{i i}$ is represented as $R_{i}$.

Then, the corresponding error dynamics equation of the integrated sensor can be obtained as shown in equation (4). The following analyses in this paper will ignore the measurement noise for the secondary sensor for simplification because of the difficulty of sampling times consistency between the primary sensor and the secondary sensor. The error equation of the integrated sensor is shown as follows:

$$
\begin{aligned}
x^{+} & =\left[\begin{array}{c}
x_{p}^{+} \\
x_{s}^{+}
\end{array}\right]=\left[\begin{array}{cc}
A_{p} & 0 \\
0 & A_{s}
\end{array}\right]\left[\begin{array}{c}
x_{p} \\
x_{s}
\end{array}\right]+\left[\begin{array}{cc}
B_{p} & 0 \\
0 & B_{s}
\end{array}\right]\left[\begin{array}{l}
w_{p} \\
w_{s}
\end{array}\right], \\
z_{i} & =z_{p i}-z_{s i} .
\end{aligned}
$$

To facilitate the following analysis, equation (5) can be derived by equation (4).

$$
\left\{\begin{array}{l}
x_{k+1}=A_{k} x_{k}+B_{k} w_{k} \\
z_{i, k+1}=H_{i, k} x_{k}+F_{i, k} v_{i, k}
\end{array}\right.
$$

where $x_{k}=\operatorname{col}\left(x_{p}, x_{s}\right)$ is the error state vector and $w_{k}=$ $\operatorname{col}\left(w_{p}, w_{s}\right)$ represents the system noise vector in the integrated error system. $v_{i}$ is the measurement noise of the primary sensor, that is, $v_{i}=v_{p i} . A_{k}=\operatorname{diag}\left(A_{p}, A_{S}\right)$ and $B_{k}=$ $\operatorname{diag}\left(B_{p}, B_{S}\right)$ are system matrices with proper dimensions. $H_{i}$ is measuring matrices. $F_{i}$ is the fault matrices. 
2.2. Event-Triggered Consensus Kalman Filter (ET-KCF). In practical applications, the sensor nodes are constrained by sensing abilities and perceived environments, etc. Uncertainties and errors exist in the same type of sensor nodes located in different positions when the sensor node acquires the local information. The packet loss has two different types. The loss of communication values happens in nodes of the wireless sensor network. Furthermore, loss of the measurement data for each node occurs in networks. The packet loss can impact the accuracy of the algorithm estimates and may even diverge estimates. From a network perspective, one of the most important indexes reflecting communication state in wireless sensor networks is the packet loss rate. The packet loss rate of the data receiving terminal in the general network environment is as high as $5 \%-10 \%$ [33]. At the same time, because the different materials and sizes of the obstacles will inevitably lead to path loss in the transmission process, it will affect the accuracy of the algorithm estimation [34]. Path loss is the magnitude of the attenuation of the signal caused by the incoming electromagnetic environment between the transmitting and receiving nodes. Undoubtedly, the existence of path loss will impact the estimation accuracy of WSN. Therefore, the estimation algorithm of the wireless sensor network must have corresponding fault tolerance characteristics when considering the impacts of packet losses and path losses.

In [20], considering the influence of packet losses and path losses on the algorithm, an event-triggered-based KCF algorithm is proposed. Due to the complexity of computation and scalability of the algorithm, the suboptimal filter of each node is designed as follows [20]:

$$
\left\{\begin{array}{l}
\widehat{x}_{i, k+1}=A_{k} \cdot \widehat{x}_{i, k}+\alpha_{k} A_{k} \cdot K_{i, k}\left(Z_{i, k}-H_{i, k} \cdot \widehat{x}_{i, k}\right)+\beta_{k} A_{k} \cdot C_{i, k} \sum_{j \in \mathcal{N}_{i}}\left[\left(1-\gamma_{i j}\right) \hat{x}_{j, k}^{o}-\widehat{x}_{i, k}\right], \\
K_{i, k}=P_{i, k} H_{i, k}^{T}\left(H_{i, k} P_{i, k} H_{i, k}^{T}+R_{i, k}\right)^{-1} \\
P_{i, k+1}=\left(A_{k}-A_{k} K_{i, k} H_{i, k}\right) P_{i, k}\left(A_{k}-A_{k} K_{i, k} H_{i, k}\right)^{T}+A_{k} K_{i, k} R_{i, k} K_{i, k}^{T} A_{k}^{T}+B_{k} Q_{i, k} B_{k}^{T} .
\end{array}\right.
$$

The algorithm will be collectively referred to as ET-KCF, and its specific content can be found in [20]. ET-KCF can effectively decrease the computational complexity of the algorithm and improve the real-time performance over WSN. At the same time, the existence of the trigger mechanism reduces the energy consumption among sensor transmissions. The consensus term of the state estimation can make all sensor nodes converge to the same estimated value. That is, each sensor node combines its measured value with information from neighbor nodes to achieve global consistent estimation, which can well help solve the problem of sensor false alarm in the cabin.

\section{Event-Triggered Kalman Consensus Filtering Algorithm under Switched Quantitative Communication}

The quantification of signals can be regarded as converting the input threshold-known signal into a finite number of discrete signals within the boundary value according to the protocol. When the signal is transmitted, only the discrete values need to be encoded and transmitted, and then transmission loads can be greatly reduced. Normally, the quantizer sets relevant initial parameters in advance, such as quantitative bits. The more bits, the more accurate. However, the quantizer becomes more complex. Therefore, the complexity and data accuracy for using a quantizer are contradictory. It is necessary to select the suitable relevant parameters of the quantizer.

3.1. Uniform Quantizer. The uniform quantizer is a linear quantizer that uniformly divides the input signal into quantized intervals according to the set quantization level. Suppose that the range of the observed signal of a node is between $[-U, U]$, where $U$ is a known parameter. The quantization level is divided according to the interval of the range of the observation signal of the sensor node. In general, the quantization level is generally divided according to the integer order of 2 , where the order is the number of quantization bits. $x$ is the state variable to be estimated. The measured value $z$ is quantized into a new value with $l$ ' bits, quantization interval length $\Delta=\left(2 U /\left(2^{l^{\prime}}-1\right)\right)$, quantification level $2^{l^{\prime}}$, output value $u_{i}=-U+\Delta i, i=(1,2, \ldots)$. Then, a uniform quantitative mathematical model can be expressed as follows:

$$
Q(z)=u_{i}, \quad z \in\left(u_{i}, u_{i+1}\right) .
$$

Lemma 1 (see [35]). Define $Q(z)$ as a quantized observation value in $z_{i, k+1}=H_{i, k} x_{k}+F_{i, k} v_{i, k}$ with quantized bits l', $Q(z) \in[-U, U]$. Then, $Q(z)$ is an unbiased estimate of state, $x$, and

$$
E\left(|Q(z)-x|^{2}\right) \leq \frac{U^{2}}{\left(2^{l^{\prime}}-1\right)^{2}}+R, \quad l^{\prime} \geq 1
$$

where $R$ is the observed noise covariance. The schematic diagram of the uniform quantization with even quantization intervals is shown in Figure 2.

It can be seen that since the uniform quantizer divides the original signal evenly, the quantization error caused by the uniform quantizer has an upper bound, and the boundary value is related to the quantization level. 


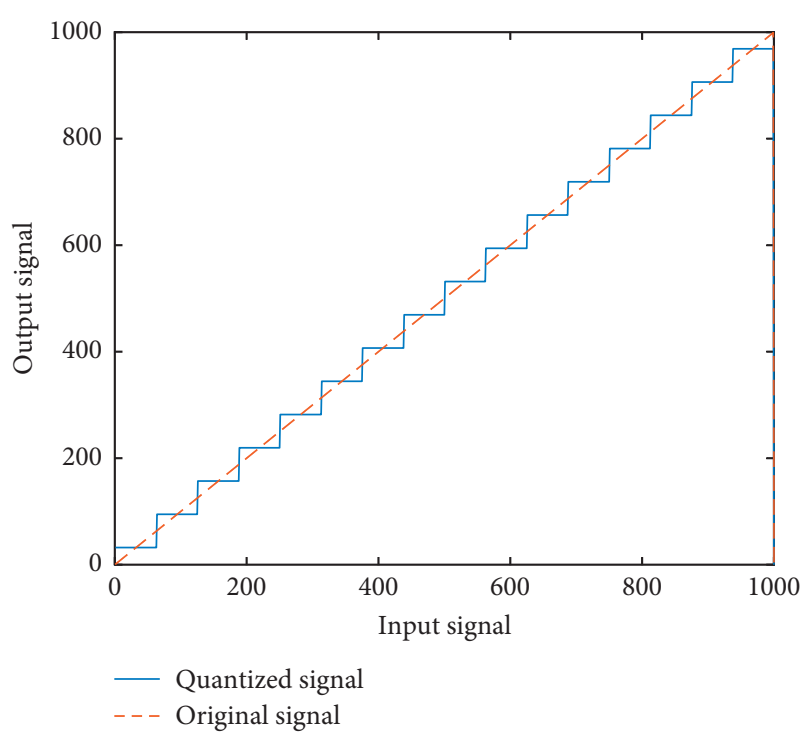

Figure 2: Schematic diagram of a uniform quantizer with even quantization intervals.

3.2. Logarithmic Quantizer. Logarithmic quantization is a nonuniform quantization strategy that divides the quantization interval into logarithmic levels. The corresponding mathematical model can be depicted as follows:

$$
Q(u)= \begin{cases}u_{i}, & \frac{u_{i}}{1+\delta}<u<\frac{u_{i}}{1-\delta}, \\ 0, & 0 \leq u<\frac{d}{1+\delta}, \\ -q(-u), & u<0,\end{cases}
$$

where $u_{i}=\rho^{(1-i)} d,(i=1,2, \ldots), \rho=((1+\delta) /(1-\delta))$ is the quantizer parameter $d>0,0<\rho<1$, and $\delta$ determines the quantization density. A schematic diagram of a logarithmic quantizer with uneven intervals is illustrated as shown in Figure 3.

If the quantization density selects logarithmic distribution and the quantized signal is large, the quantization error will be large and will impact the system performance, even causing the system to be unstable. If the quantized signal is a small signal, such as a small estimated error that fluctuates near the origin, the output signal of the logarithm quantizer is more accurate.

The uniform quantizer can directly quantize the original information, and the quantization error has good statistical characteristics. Therefore, it is suitable for the quantization of the original measurement signal. The logarithmic quantizer uses a nonlinear quantization interval division method, which can ensure that the quantized information has good precision. So it is more suitable for quantifying error signals instead of the original signals. According to the comparison between the uniform quantizer and the logarithm quantizer, these two algorithm structures are easy to implement because they are both static quantizers and only need to determine the boundary value of the input signal and

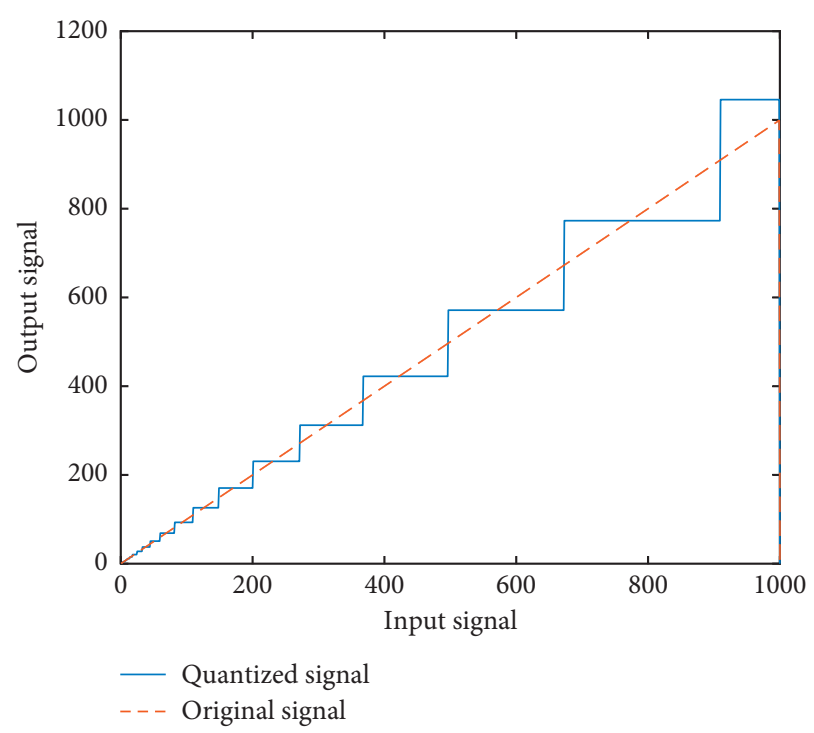

FIGURE 3: The schematics of a logarithmic quantizer with uneven intervals.

the division of the quantization interval. Therefore, both quantizers can be combined and used in practical engineering, such as state estimations of cabin pollutant concentrations.

\subsection{Event-Triggered Kalman Consensus Filtering Algorithm} under Switched Quantitative Communication. To reduce the burden of signal transmission bandwidth, it is necessary to quantify the observation values transmitted from the observer to the estimator and the estimated values among nodes. The state estimation algorithm used in this paper employs the distributed ET-KCF integrated sensor structure when considering the inevitable packet loss phenomena in the actual environment. The observed value is the error of the observation values between the primary and secondary sensors, respectively. If packet loss of any sensor for each node happens, the error amount becomes close to the observation value from another sensor. Furthermore, the quantized error will correspondingly be large, affecting estimation accuracy. Therefore, this article proposes a novel switched quantizer that includes the properties of logarithmic quantization and uniform quantization. The expression is shown as follows:

$$
q(u)= \begin{cases}\rho^{i} d, & 0<u \leq u_{\mathrm{th}}, \\ u_{t h}+\Delta_{q}\left(i+1-2^{l^{\prime}}\right), & u>u_{\mathrm{th}}, \\ -q(-u), & u<0,\end{cases}
$$

where parameters $\rho, d$ satisfy $\left(\left(\rho^{l^{\prime}-2} d\right) /(1-\delta)\right)-\left(\left(\rho^{l^{\prime}-2} d\right) /\right.$ $(1+\delta))<\Delta_{q} \quad$ and $\quad u_{\text {th }} \in\left(\left(\left(\rho^{l^{\prime}-1} d\right) /(1+\delta)\right), \quad\left(\left(\rho^{l^{\prime}-1} d\right) /\right.\right.$ $\left.(1+\delta))+\Delta_{q}\right)$.

It can be seen that the selection of parameters $\rho, d$ ensures that the quantization error of the designed switched quantizer does not exceed the quantization error, $\Delta_{q}$ of the uniform quantizer, and the selection of the threshold $u_{\text {th }}$ is 
related to $\Delta_{q}$. When there is no packet loss, the first half of the logarithm quantizer can ensure that it has a high quantization precision since the error is the amount that fluctuates around the origin. However, when the packet loss occurs, the input amount becomes biased. The uniform quantization in the latter half can ensure that the quantization error of the entire quantizer is less than $\Delta_{q}$. The schematic diagram between input and output signals for the switched quantizer proposed in this paper is shown in Figure 4.

Therefore, this paper studies a switched quantizer-based event-triggered Kalman consensus filter (QEF-KCF). Figure 5 shows a schematic diagram of a node over the distributed integrated network with switched quantizers. As Figure 5 shows, the quantizer is used between the observer and estimator. That is, the estimator receives the quantized signals. Another quantizer is also applied behind the estimator. Then, the quantized output of the estimator was simultaneously transmitted to the neighbor nodes. Each quantizer is a switched quantizer. ET-KCF algorithm is employed to estimate the state values.

The specific steps of the QET-KCF estimation algorithm are listed as follows, $i$ denotes the $i$-th sensor:

Step 1. Initialize the state and error covariance matrices.

$$
\begin{aligned}
& P_{i}=P_{0}, \\
& \widehat{x}_{i}=x_{0} .
\end{aligned}
$$

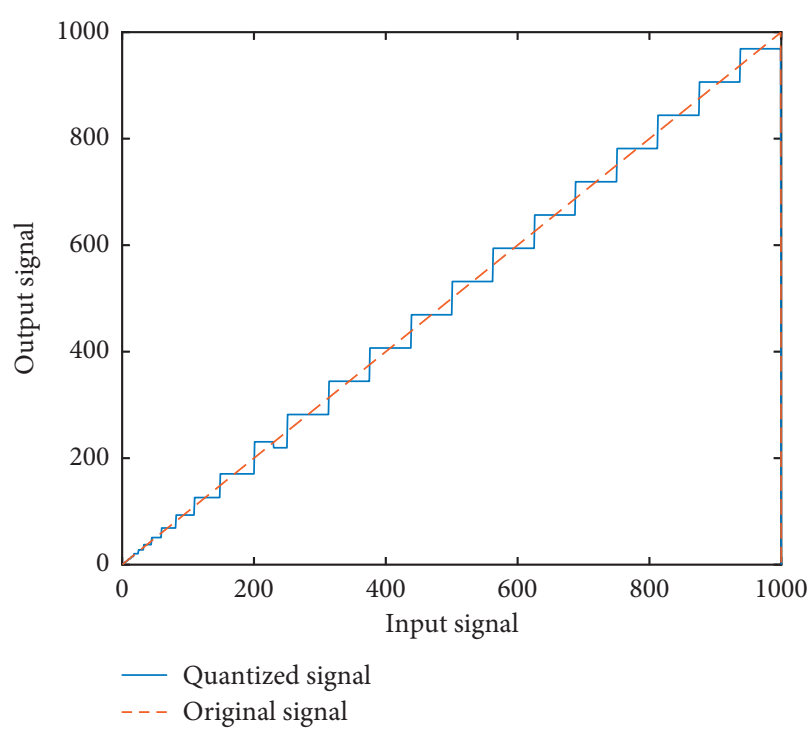

Figure 4: The schematics of a switched quantizer.

Step 2. Quantize the measured values from neighbor sensors.

$$
z=Q(z)
$$

Step 3. Calculate the estimated values by the estimator.

$$
\widehat{x}_{i, k+1}=A_{k} \cdot \widehat{x}_{i, k}+\alpha_{k} A_{k} \cdot K_{i, k}\left[Q\left(Z_{i, k}\right)-H_{i, k} \cdot \widehat{x}_{i, k}\right]+\beta_{k} A_{k} \cdot C_{i, k} \sum_{j \in \mathcal{N}_{i}}\left[\left(1-\gamma_{i j}\right) Q\left(\hat{x}_{j, k}^{o}\right)-\widehat{x}_{i, k}\right],
$$

where $\gamma_{i j}$ indicates the path loss rate between node $i$ and node $j, \widehat{x}_{j}^{o}$ is the broadcast estimate at the current time, $\alpha_{k}$ is a Bernoulli distributed sequence with a value 0 , or 1 and satisfies $P\left\{\alpha_{k}=1\right\}=\mu, C_{i, k}$ is the consensus gain matrices.

Step 4. Determine whether triggered conditions are energized or not by the following function:

$$
\widehat{x}_{i, k+1}= \begin{cases}\hat{x}_{i, k+1}, & \varphi_{i}>0 \\ \hat{x}_{i, k}^{o}, & \varphi_{i} \leq 0\end{cases}
$$

where $\varphi_{i}=\left(\hat{x}_{i, k}^{o}-\hat{x}_{i, k}\right)^{T}\left(\hat{x}_{i, k}^{o}-\widehat{x}_{i, k}\right)-g_{i}, \hat{x}_{i, k}^{o}$ is the broadcasted estimate at the previous time instant, and $g_{i}$ is the triggered threshold and positive. If $\varphi_{i}>0$, trigger the event, then transmit current estimated values. Otherwise, use previous values. In this case, no transmissions occur and energy is saved.

Step 5. Quantize the estimated values and transmit them to neighbors.

$$
\hat{x}_{i, k+1}=Q\left(\hat{x}_{i, k+1}\right) \text {. }
$$

Step 6. Update the gain matrices and error covariance matrices.

$$
\begin{aligned}
K_{i, k}= & P_{i, k} H_{i, k}^{T}\left[H_{i, k} P_{i, k} H_{i, k}^{T}+\left(R_{i, k}+\Delta_{q}^{2}\right)\right]^{-1}, \\
P_{i, k+1}= & \left(A_{k}-A_{k} K_{i, k} H_{i, k}\right) P_{i, k}\left(A_{k}-A_{k} K_{i, k} H_{i, k}\right)^{T}+A_{k} K_{i, k}\left(R_{i, k}+\Delta_{q}^{2}\right) K_{i, k}^{T} A_{k}^{T} \\
& +B_{k}\left(Q_{i, k}+\Delta_{q}^{2}\right) B_{k}^{T} .
\end{aligned}
$$




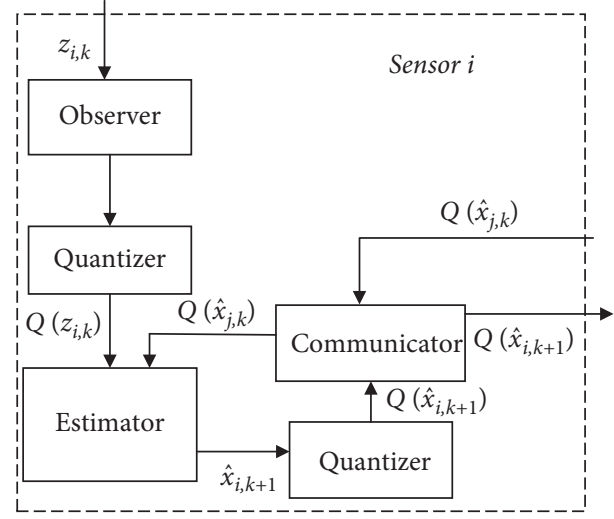

FIGURE 5: Schematic diagram of a node over a distributed integrated network.

Step 7. Calculate real-time assessment for different environment parameters.

$$
Z_{O i}=Z_{P i}-\widehat{x}_{i} .
$$

If any environment parameter is greater than the corresponding safe threshold, $Z_{\mathrm{O} i} \geq Z_{b l}$, then alarm. Otherwise, output air quality index AQI,

$$
\mathrm{AQI}=\sum_{l} \frac{\alpha_{l}\left(Z_{b l}-Z_{O i}\right)}{\left\|Z_{b l}\right\|},
$$

where $Z_{b l}$ is the safe threshold for a certain pollutant, $l$, and $\alpha_{l}$ is weighted value for the corresponding pollutant.

Step 8. Return to Step 2 and cycle through.

After inserting quantizers in sensor networks, as long as the number of bits of quantizers and the range of the quantization interval are specified in advance, the binary digits can be used instead of the analogical value for state estimation to communicate among nodes. Therefore, the bandwidth requirement of communication is effectively reduced. The performance of the quantized algorithm will be demonstrated by simulation verifications in Section 4 by comparing it to the ET-KCF algorithm proposed in [20].

3.4. Stability Analysis for Switched Quantitative ET-KCT Algorithm. As illustrated in Figure 5, one quantizer is located between observer and estimator, and the other one is placed behind the estimator. It is known that the quantization error is smaller than $\Delta_{q}$. Therefore, the observation noise should also consider the quantization error. That is, the observation noise covariance matrix becomes $R^{\prime}=R+\Delta_{q}^{2}$.
Furthermore, to analyze the stability of the switched QET$\mathrm{KCF}$, some assumptions are set as follows.

Assumption 1. Matrix $A_{k}$ is the nonsingular for each $k>0$.

Assumption 2. $\left(A_{k}, H_{i, k}\right)$ is uniformly observable.

Assumption 3. The system and parameter matrices are bounded by the positive scalars $\bar{a}, \bar{b}, \bar{h}, q, \underline{r}, \bar{d}, p, \bar{p}$ :

$$
\begin{aligned}
\left\|A_{k}\right\| & \leq \bar{a}, \\
\left\|B_{k}\right\| & \leq \bar{b}, \\
\left\|H_{i, k}\right\| & \leq \bar{h}, \\
Q_{k} & \geq \underline{q} I, \\
R_{i, k}^{\prime} & \geq \underline{r} I, \\
\left\|F_{i, k}\right\| & \leq \bar{d}, \\
\underline{p}_{i} I & \leq P_{i, k} \leq \bar{p}_{i} I,
\end{aligned}
$$

where $I$ is the identity matrix.

According to [36], if the matrix $\left(A_{k}, H_{i, k}\right)$ meets the uniform observable condition for each time constant $k$ in the system (5), $P_{i, k}$ has upper and lower bounds.

Lemma 2 ([36], Lemma 2.1). Assume there is a stochastic process $V_{k}\left(\xi_{k}\right)$ and real numbers $\underline{v}, \bar{v}, l>0$ and $0 \leq \alpha \leq 1$ such that

$$
\begin{aligned}
\underline{v}\left\|\xi_{k}\right\|^{2} & \leq V_{k}\left(\xi_{k}\right) \leq \bar{v}\left\|\xi_{k}\right\|^{2}, \\
E\left\{V_{k}\left(\xi_{k}\right) \mid \xi_{k-1}\right\} & \leq(1-\alpha) V_{k-1}\left(\xi_{k-1}\right)+l .
\end{aligned}
$$

Then, the stochastic process is exponentially bounded in the mean square, such that

$$
E\left\{\left\|\xi_{k}\right\|^{2}\right\} \leq \frac{\bar{v}}{\underline{v}} E\left\{\left\|\xi_{0}\right\|^{2}\right\}(1-\alpha)^{k}+\frac{l}{\underline{v}} \sum_{i=1}^{k}(1-\alpha)^{i},
$$

and the stochastic process is bounded with probability 1 .

In the following analyses, Lemma 1 will be used as the boundary condition for the estimation process of QET-KCF. The boundaries of these parameters for each sensor node are obtained as described by Lemmas 3 and 4 .

Lemma 3 (see [36], Lemma 3.1). Based on Assumption 3, there exists a real number, $0<\kappa_{i}<1(i=1,2, \ldots, N)$, making the following equation works:

$$
\left(A_{k}-\mu A_{k} \cdot K_{i, k} \cdot H_{i, k}\right)^{T} \cdot P_{i, k+1}^{-1}\left(A_{k}-\mu A_{k} \cdot K_{i, k} \cdot H_{i, k}\right) \leq\left(1-\kappa_{i}\right) P_{i, k}^{-1}
$$

where 


$$
\kappa_{i}=1-\frac{1}{1+\left(\underline{q} / \bar{p}\left(\left(\bar{a}+\mu \cdot \bar{a} \cdot \bar{p} \cdot \bar{h}^{2}\right) / \underline{r}\right)^{2}\right)} .
$$

Lemma 4 (see [36], Lemma 3.3). Under Assumption 3, there is a real number $\epsilon_{i}>0(i=1,2, \ldots, N)$ such that

$$
E\left\{\begin{array}{c}
\mu^{2} v_{i, k}^{T} \cdot F_{i, k}^{T} \cdot K_{i, k}^{T} \cdot A_{k}^{T} \cdot P_{i, k+1}^{-1} \cdot A_{k} \cdot K_{i, k} F_{i, k} \cdot v_{i, k}+ \\
w^{T} \cdot B^{T} \cdot P_{i, k+1}^{-1} \cdot B \cdot w
\end{array}\right\} \leq \varepsilon_{i} .
$$

In the following part, the analysis results of stability are discussed.

Theorem 1. According to Assumptions 1-3, an estimation error of a discrete linear time-varying system (5) with QETKCF is exponentially bounded in the mean square with probability 1 when the initial prediction error is bounded.

The proving process of Theorem 1 is represented in the appendix.

The upper bound of $\sigma$ for the consensus gain, $\sigma^{*}$, is

$$
\begin{aligned}
\sigma^{*} & =\frac{-\bar{p}\left(\beta_{1}+\beta_{2}\right)+\theta}{2 \mu_{2}\left(\bar{p} \beta_{1}^{2}+\bar{p} \beta_{1}^{2}\left(1-m^{\prime}\right) /\left(\underline{p}+\bar{p} \beta_{1} \beta_{2}\right)\right) \lambda_{\max }\left(\mathscr{L}^{T} \mathscr{L}\right)} \\
\theta & =\left[\bar{p}^{2}\left(\beta_{1}+\beta_{2}\right)^{2}+4\left(\frac{\bar{p} \beta_{1}^{2}+\bar{p} \beta_{1}^{2}\left(1-m^{\prime}\right)}{\underline{p}+\bar{p} \beta_{1} \beta_{2}}\right) \lambda_{\max }\left(\mathscr{L}^{T} \mathscr{L}\right) m^{\prime}\right]^{1 / 2} .
\end{aligned}
$$

Remark 1. It is noticed that compared to the upper bound value presented in [19], the upper limit $\sigma^{*}$ becomes smaller in this paper. It is known that the observation error covariance matrix becomes $R^{\prime}=R+\Delta_{q}^{2}$ due to the introduction of quantization error. This leads $m$ in [20] to $m$ ' in this paper, where $m^{\prime}=\min \left\{\kappa_{1}, \ldots, \kappa_{N}\right\}$; therefore, the upper bound limit decreases.

\section{Performance Simulations for the Switched ET-KCF Algorithm}

The mean estimation error (MEE) and mean consensus error (MCE) defined in [31] are used as the performance indexes. The simulation is performed with the Monte Carlo method on the Matlab platform. The statistical averages are used to analyze the error responses of the wireless sensor monitoring system.

Mean estimation error (MEE) is

$$
\mathrm{MEE}_{k}=\sqrt{\frac{\sum_{i=1}^{N}\left(e_{i, k}^{T} e_{i, k}\right)}{N}}
$$

where $e_{i, k}=x_{i, k}-\hat{x}_{i, k}$.

Mean consensus error (MCE) is

$$
\mathrm{MCE}_{k}=\sqrt{\frac{\sum_{i=1}^{N}\left(\delta_{i, k}^{T} \delta_{i, k}\right)}{N}}
$$

where $\delta_{i, k}=\widehat{x}_{i, k}-\left(\sum_{i=1}^{N} \hat{x}_{i, k} / N\right), k$ is defined as the time instant, and $N$ is the node number.

This article does not provide the approach to get the dynamic equations of integrated sensors in detail. As illustrated in [20], there are several ways to derive the models such as the theoretical analysis of the measurement error and identification of practical measurement values. In order to be convenient to compare the proposed method to the previous works in [20], this paper selects the same dynamic models for the 10 nodes over the distributed monitoring integrated sensor network illustrated in Figure 6 and conducts simulation validations.

Suppose that the measurement error dynamics of an integrated sensor is as follows:

$$
\begin{aligned}
x_{i, k+1}= & {\left[\begin{array}{cc}
1+0.005 \cdot \sin (j) & -0.002 \cdot \cos (j)+0.0001 \cdot \text { rand } \\
0.002 \cdot \cos (j)+0.0001 \cdot \text { rand } & 1+0.005 \cdot \sin (j)
\end{array}\right] x_{i, k}+\left[\begin{array}{cc}
0.5 & 0 \\
0 & 0.5
\end{array}\right] w_{k}, } \\
z_{i, k+1}= & {\left[\begin{array}{cc}
1+0.005 \cdot \cos (j) & -0.002 \cdot \sin (j)+0.0001 \cdot \text { rand } \\
0.002 \cdot \sin (j)+0.0001 \cdot \text { rand } & 1+0.005 \cdot \cos (j)
\end{array}\right] x_{i, k} } \\
& +\left[\begin{array}{cc}
1+0.5 \cdot \cos (j) & -0.02 \cdot \sin (j)+0.001 \cdot \operatorname{rand} \\
0.02 \cdot \sin (j)+0.001 \cdot \text { rand } & 1+0.5 \cdot \cos (j)
\end{array}\right] v_{p i} .
\end{aligned}
$$




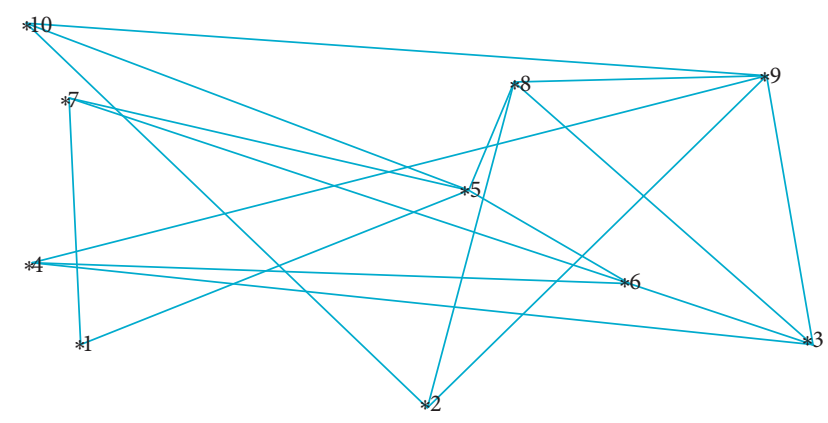

FIGURE 6: The topological graph of a distributed monitoring sensor network.

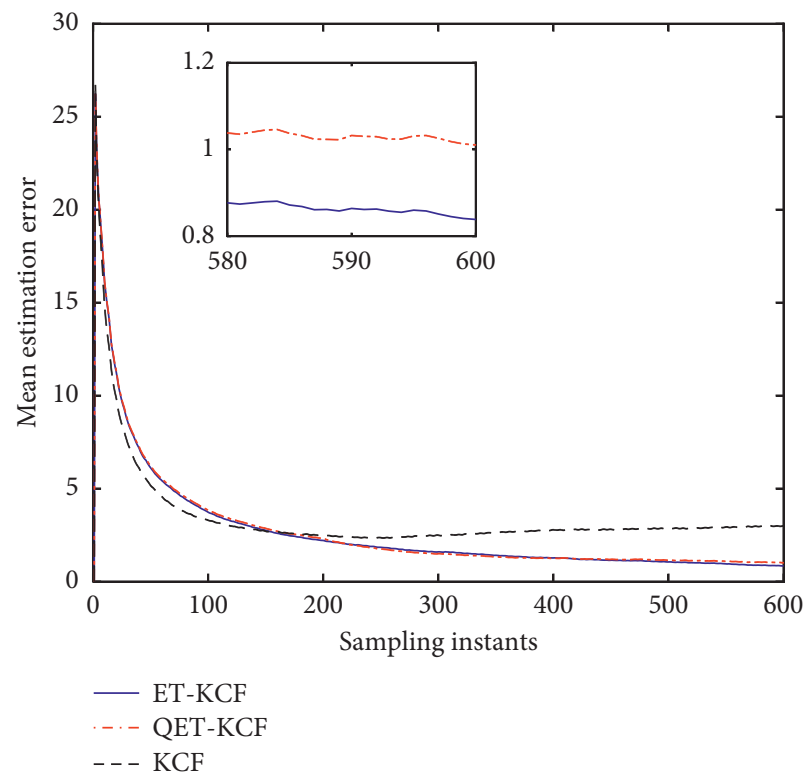

Figure 7: Comparison of mean estimation errors among different filters in the event of packet and path losses.

In this example, also assume the initial measurement error $x_{0}=(8,12)^{T}$, the initial prediction error matrix $P_{0}=10 I_{2}$. The network and the measurement noises are described by independent Gaussian white noises with covariance 10 and $100 i$, respectively. Let the path loss rate $\gamma_{i j}$ between nodes $i$ and $j$ be 0.05 . The sampling duration is $5 \mathrm{~ms}$. And the observed packet loss and the communication packet loss rates are 0.2 and $\mu_{1}=\mu_{2}=0.8$ respectively. The quantizer parameters, $\delta$ and $d$ are set to be 0.05 and 1 . The number of quantization bits is $l=4$.

As seen from Figure 7, when packet losses and path losses exist, KCF diverges. However, ET-KCF and switched QET-KCF can still converge stably. However, the accuracy of the switched QET-KCF is slightly reduced. This is because the quantization error is introduced by inserting the quantizers. The unquantized algorithm is developed based on the ideal condition without the transmission bandwidth limitation in each state estimation. Therefore, the accuracy is better. However, the quantized algorithm can effectively reduce the demand for communication bandwidth while

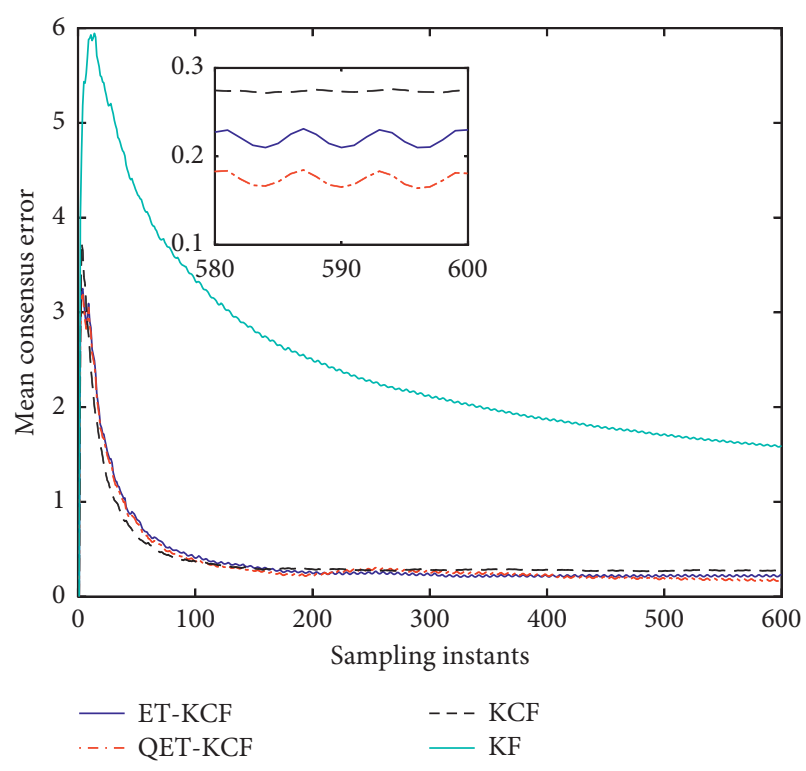

FIGURE 8: Comparison of mean consensus errors among different filters in the event of packet and path losses.

only sacrificing the accuracy slightly. Therefore, the advantages of the introduction of quantizers can overwhelm the disadvantages.

As seen from Figure 8, the mean consensus error of the switched quantized QET-KCF has the same order as ET$\mathrm{KCF}$ and $\mathrm{KCF}$ and is closed to them. Furthermore, it is much less than that of the local Kalman filter algorithm in the event of packet and path losses.

It can be concluded that the performance of the algorithm by adding the quantizers is not greatly affected. The estimation error still converges to the finite value very quickly, and the consensus error still maintains a low level which means the estimated values among nodes tend to be consistent. Simultaneously, the burdens of communications have been improved greatly.

\section{Conclusion}

This paper designs a new switched quantizer and applies the new quantizers in ET-KCF to form a switched QET-KCF algorithm that is employed in cabin environment parameter estimations. The proposed quantizer can ensure that the quantization error is bounded. The corresponding stability analysis is theoretically proved. The simulation results show that the proposed algorithm in this paper can accurately and timely estimate parameters and effectively reduce the burdens of network communication bandwidths. This is a promising method to solve the communication burden issue in cabin WSN in the future.

\section{Appendix}

Proof. The prediction error is defined as $e_{k}=\left[e_{1}^{T}, \ldots, e_{N}^{T}\right]^{T}$. $P=\operatorname{diag}\left\{P_{1}, \ldots, P_{N}\right\}$ is the corresponding block diagonal matrix. The following Lyapunov function is constructed: 


$$
V_{k}\left(e_{k}\right)=e_{k}^{T} P^{-1} e_{k}=\sum_{i=1}^{N} e_{i, k}^{T} P_{i, k}^{-1} e_{i, k} .
$$

As mentioned in Assumption 2, $\underline{p}_{i} I \leq P_{i, k} \leq \bar{p}_{i} I$; then,

$$
\frac{1}{\bar{p}}\left\|e_{k}\right\|^{2} \leq V_{k}\left(e_{k}\right) \leq \frac{1}{\underline{p}}\left\|e_{k}\right\|^{2},
$$

where $\bar{p}=\max \left\{\bar{p}_{1}, \ldots, \bar{p}_{N}\right\}$ and $\underline{p}=\max \left\{\underline{p}_{1}, \ldots, \underline{p}_{N}\right\}$.

Inequality (A.2) satisfies Lemma 1. And we have to discuss the expectation value of the Lyapunov function $E\left\{V_{k+1}\left(e_{k+1}\right)\right\}$ of next time instant to prove that exponential boundedness of the process $e$. Based on the definition of $e_{i, k}$, the prediction error dynamics of $e_{i, k+1}$ are defined as follows:

$$
\begin{aligned}
e_{i, k+1}= & \left(A_{k}-\alpha_{k} A_{k} \cdot K_{i, k} \cdot H_{i, k}\right) \cdot e_{i, k}+\alpha_{k} A_{k} \cdot K_{i, k} F_{i, k} \cdot v_{i, k} \\
& +\beta_{k} A_{k} \cdot C_{i, k} \sum_{j \in \mathcal{N}_{i}}\left(1-\gamma_{i j}\right) \widetilde{e}_{j, k}+\beta_{k} A_{k} \cdot C_{i, k} \sum_{j \in \mathcal{N}_{i}}\left(1-\gamma_{i j}\right)\left(e_{j, k}-e_{i, k}\right) \\
& -\beta_{k} A_{k} \cdot C_{i, k} \sum_{j \in \mathcal{N}_{i}} \gamma_{i j} \widehat{x}_{i, k}+\beta_{k} A_{k} \cdot C_{i, k} \sum_{j \in \mathcal{N}_{i}}\left(1-\gamma_{i j}\right) \Delta q-B_{k} w_{k} .
\end{aligned}
$$

According to the definition of the Lyapunov function, we have the following:

$$
\begin{aligned}
E\left\{V_{k+1}\left(e_{k+1}\right)\right\}= & \sum_{i=1}^{N} e_{i, k}^{T}\left(A_{k}-\mu_{1} A_{k} \cdot K_{i, k} \cdot H_{i, k}\right)^{T} \cdot P_{i, k+1}^{-1} \cdot\left(A_{k}-\mu_{1} A_{k} \cdot K_{i, k} \cdot H_{i, k}\right) e_{i, k} \\
& +2 \mu_{2} \sum_{i=1}^{N} \sum_{j \in \mathcal{N}_{i}}\left(1-\gamma_{i j}\right) e_{i, k}^{T}\left(A_{k}-\mu_{1} A_{k} \cdot K_{i, k} \cdot H_{i, k}\right)^{T} \cdot P_{i, k+1}^{-1} \cdot A_{k} \cdot C_{i, k} \widetilde{e}_{j, k} \\
& +2 \mu_{2} \sum_{i=1}^{N} \sum_{j \in \mathcal{N}_{i}}\left(1-\gamma_{i j}\right) e_{i, k}^{T}\left(A_{k}-\mu_{1} A_{k} \cdot K_{i, k} \cdot H_{i, k}\right)^{T} \cdot P_{i, k+1}^{-1} \cdot A_{k} \cdot C_{i, k}\left(e_{j, k}-e_{i, k}\right) \\
& -2 \mu_{2} \sum_{i=1}^{N} \sum_{j \in \mathcal{N}_{i}} \gamma_{i j} e_{i, k}^{T}\left(A_{k}-\mu_{1} A_{k} \cdot K_{i, k} \cdot H_{i, k}\right)^{T} \cdot P_{i, k+1}^{-1} \cdot A_{k} \cdot C_{i, k} \widehat{x}_{i, k} \\
& +\sum_{i=1}^{N} \mu_{1}^{2} v_{i, k}^{T} \cdot F_{i, k}^{T} \cdot K_{i, k}^{T} \cdot A_{k}^{T} \cdot P_{i, k+1}^{-1} \cdot A_{k} \cdot K_{i, k} \cdot F_{i, k} \cdot v_{i, k} \\
& +\mu_{2}^{2} \sum_{i=1}^{N} \sum_{j \in \mathcal{N}_{i}}\left(1-\gamma_{i j}\right)^{2} \widetilde{e}_{j, k}^{T} C_{i, k}^{T} \cdot A_{k}^{T} \cdot P_{i, k+1}^{-1} \cdot A_{k} \cdot C_{i, k} \widetilde{e}_{j, k} \\
& +2 \mu_{2}^{2} \sum_{i=1}^{N} \sum_{j \in \mathcal{N}_{i}}\left(1-\gamma_{i j}\right)^{2} \widetilde{e}_{j, k}^{T} C_{i, k}^{T} \cdot A_{k}^{T} \cdot P_{i, k+1}^{-1} \cdot A_{k} \cdot C_{i, k}\left(e_{j, k}-e_{i, k}\right) \\
& -2 \mu_{2}^{2} \sum_{i=1}^{N} \sum_{j \in \mathcal{N}_{i}}\left(1-\gamma_{i j}\right) \gamma_{i j} \tilde{e}_{j, k}^{T} C_{i, k}^{T} \cdot A_{k}^{T} \cdot P_{i, k+1}^{-1} \cdot A_{k} \cdot C_{i, k} \widehat{x}_{i, k} \\
& +\mu_{2}^{2} \sum_{i=1}^{N} \sum_{j \in \mathcal{N}_{i}}\left(1-\gamma_{i j}\right)^{2}\left(e_{j, k}-e_{i, k}\right)^{T} C_{i, k}^{T} \cdot A_{k}^{T} \cdot P_{i, k+1}^{-1} \cdot A_{k} \cdot C_{i, k}\left(e_{j, k}-e_{i, k}\right) \\
& -2 \mu_{2}^{2} \sum_{i=1}^{N} \sum_{j \in \mathcal{N}_{i}}\left(1-\gamma_{i j}\right) \gamma_{i j}\left(e_{j, k}-e_{i, k}\right)^{T} C_{i, k}^{T} \cdot A_{k}^{T} \cdot P_{i, k+1}^{-1} \cdot A_{k} \cdot C_{i, k} \widehat{x}_{i, k} \\
& +\mu_{2}^{2} \sum_{i=1}^{N} \sum_{j \in \mathcal{N}_{i}} \gamma_{i j}^{2} \widehat{x}_{i, k}^{T} C_{i, k}^{T} \cdot A_{k}^{T} \cdot P_{i, k+1}^{-1} \cdot A_{k} \cdot C_{i, k} \widehat{x}_{i, k} \\
& +\mu_{2}^{2} \sum_{i=1}^{N} \sum_{j \in \mathcal{N}_{i}}\left(1-\gamma_{i j}\right)^{2} \widehat{x}_{i, k}^{T} \Delta_{q}^{T} \cdot C_{i, k}^{T} \cdot A_{k}^{T} \cdot P_{i, k+1}^{-1} \cdot A_{k} \cdot C_{i, k} \Delta_{q} \\
& +\sum_{i=1}^{N} w_{k}^{T} \cdot B_{k}^{T} \cdot P_{i, k+1}^{-1} \cdot B_{k} \cdot w_{k} \cdot \\
& \\
&
\end{aligned}
$$




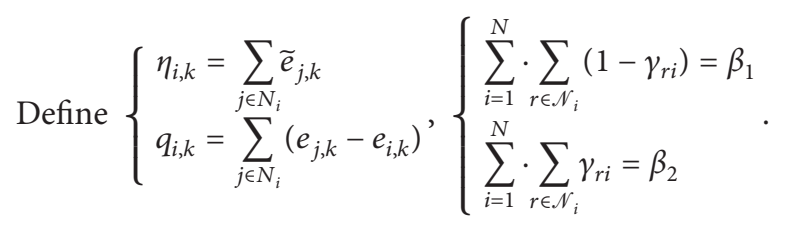

To reduce the complexity of analysis, let the consensus gain be as follows:

$$
\begin{aligned}
& C_{i, k}=\sigma\left[\left(A_{k}-\mu A_{k} \cdot K_{i, k} \cdot H_{i, k}\right)^{T} \cdot P_{i, k+1}^{-1} \cdot A_{k}\right]^{-1} \Lambda_{i, k}, \\
& \Lambda_{i, k}=\left(A_{k}-\mu A_{k} \cdot K_{i, k} \cdot H_{i, k}\right)^{T} \cdot P_{i, k+1}^{-1} \cdot\left(A_{k}-\mu A_{k} \cdot K_{i, k} \cdot H_{i, k}\right) .
\end{aligned}
$$

Then,

Therefore,

$$
\begin{aligned}
C_{i, k}^{T} \cdot A_{k}^{T} \cdot P_{i, k+1}^{-1} \cdot\left(A_{k}-\mu A_{k} \cdot K_{i, k} \cdot H_{i, k}\right) & =\sigma \cdot \Lambda_{i, k}, \\
C_{i, k}^{T} \cdot A_{k}^{T} \cdot P_{i, k+1}^{-1} \cdot A_{k} \cdot C_{i, k} & =\sigma^{2} \cdot \Lambda_{i, k} .
\end{aligned}
$$

where

$$
\begin{aligned}
E\left\{V_{k+1}\left(e_{k+1}\right)\right\} \leq & \sum_{i=1}^{N} \varepsilon_{i}+\sum_{i=1}^{N}\left(1-\kappa_{i}\right) e_{i, k}^{T} \cdot P_{i, k}^{-1} \cdot e_{i, k} \\
& +2 \mu_{2}\left(\beta_{1} \sigma e_{k}^{T} \Lambda_{k} \eta_{k}+2 \beta_{1} \sigma e_{k}^{T} \Lambda_{k} q_{k}-2 \beta_{2} \sigma e_{k}^{T} \Lambda_{k} \widehat{x}_{k}\right) \\
& +\mu_{2}^{2}\left(\beta_{1}^{2} \sigma^{2} q_{k}^{T} \Lambda_{k} q_{k}-2 \beta_{1} \beta_{2} \sigma^{2} q_{k}^{T} \Lambda_{k} \widehat{x}_{k}+\beta_{2}^{2} \sigma^{2} \widehat{x}_{k}^{T} \Lambda_{k} \widehat{x}_{k}+\beta_{1}^{2} \sigma^{2} \Delta_{q}^{T} \Lambda_{k} \Delta_{q}\right), \\
& \beta_{1}^{2} \sigma^{2} \eta_{k}^{T} \Lambda_{k} \eta_{k} \leq \beta_{1}^{2} \sigma^{2} \lambda_{\max }\left(\mathscr{A}^{T} \mathscr{A}\right) \lambda_{\max }\left(\Lambda_{k}\right) \Delta .
\end{aligned}
$$

$$
\begin{aligned}
\eta_{k} & =\mathscr{A} \cdot \widetilde{e}_{k}, \\
\mathscr{A} & =E \otimes I, \\
q_{k} & =-\mathscr{L} \cdot e_{k}, \\
\mathscr{L} & =\left[\left(1-\gamma_{r}\right) D-E\right] \otimes I, \\
\eta_{k}^{T} \eta_{k} & =\widetilde{e}_{k}^{T} \mathscr{A}^{T} \mathscr{A}_{\tilde{e}_{k}} \leq \lambda_{\max }\left(\mathscr{A}^{T} \mathscr{A}\right) \Delta,
\end{aligned}
$$

For the seventh term,

$$
2 \beta_{1}^{2} \sigma^{2} \eta_{k}^{T} \Lambda_{k} q_{k} \leq \beta_{1}^{2} \sigma^{2}\left(\eta_{k}^{T} \Lambda_{k}^{T} \Lambda_{k} \eta_{k}+e_{k}^{T} \mathscr{L}^{T} \mathscr{L} e_{k}\right) .
$$

For the eighth term,

$$
\begin{aligned}
-2 \beta_{1} \beta_{2} \sigma^{2} \eta_{k}^{T} \Lambda_{k} \widehat{x}_{k} & \leq \beta_{1} \beta_{2} \sigma^{2}\left(\eta_{k}^{T} \eta_{k}+\widehat{x}_{k}^{T} \Lambda_{k}^{T} \Lambda_{k} \widehat{x}_{k}\right) \\
& \leq \beta_{1} \beta_{2} \sigma^{2} \lambda_{\max }\left(\mathscr{A}^{T} \mathscr{A}\right) \Delta+\beta_{1} \beta_{2} \sigma^{2} \lambda_{\max }\left(\Lambda_{k}^{T} \Lambda_{k}\right) \widehat{x}_{k}^{T} \widehat{x}_{k} .
\end{aligned}
$$

and $\Delta=\sum_{i=1}^{N} \delta_{i}$.

$E$ and $D$ are the adjacency matrix and degree matrix of graph $G$, respectively.

And,

$$
\begin{aligned}
+2 \beta_{1} \sigma e_{k}^{T} \Lambda_{k} \eta_{k} & \leq \beta_{1} \sigma\left(\eta_{k}^{T} \Lambda_{k}^{T} \Lambda_{k} \eta_{k}+e_{k}^{T} e_{k}\right) \\
& \leq \beta_{1} \sigma\left(\lambda_{\max }\left(\Lambda_{k}^{T} \Lambda_{k}\right) \lambda_{\max }\left(\mathscr{A}^{T} \mathscr{A}\right) \Delta\right)+\beta_{1} \sigma e_{k}^{T} e_{k} .
\end{aligned}
$$

For the fourth term,

$$
2 \beta_{1} \sigma e_{k}^{T} \Lambda_{k} q_{k} \leq-2 \sigma \lambda_{\min }\left(\Lambda_{k}\right) e_{k}^{T} \mathscr{L} e_{k} \leq 0 .
$$

For the fifth term,

$$
\begin{aligned}
-2 \beta_{2} \sigma e_{k}^{T} \Lambda_{k} \widehat{x}_{k} & \leq \beta_{2} \sigma\left(e_{k}^{T} e_{k}+\widehat{x}_{k}^{T} \Lambda_{k}^{T} \Lambda_{k} \widehat{x}_{k}\right) \\
& \leq \beta_{2} \sigma e_{k}^{T} e_{k}+\beta_{2} \sigma \lambda_{\max }\left(\Lambda_{k}^{T} \Lambda_{k}\right) \widehat{x}_{k}^{T} \widehat{x}_{k} .
\end{aligned}
$$

For the sixth term,

For the ninth term,

$\beta_{1}^{2} \sigma^{2} q_{k}^{T} \Lambda_{k} q_{k} \leq \beta_{1}^{2} \sigma^{2} \lambda_{\max }\left(\Lambda_{k}\right) \lambda_{\max }\left(\mathscr{L}^{T} \mathscr{L}\right) e_{k}^{T} e_{k}$.

For the tenth term,

$$
\begin{aligned}
-2 \beta_{1} \beta_{2} \sigma^{2} q_{k}^{T} \Lambda_{k} \widehat{x}_{k} \leq & \beta_{1} \beta_{2} \sigma^{2}\left(q_{k}^{T} q_{k}+\widehat{x}_{k}^{T} \Lambda_{k}^{T} \Lambda_{k} \widehat{x}_{k}\right) \\
\leq & \beta_{1} \beta_{2} \sigma^{2} \lambda_{\max }\left(\mathscr{L}^{T} \mathscr{L}\right) e_{k}^{T} e_{k} \\
& +\beta_{1} \beta_{2} \sigma^{2} \lambda_{\max }\left(\Lambda_{k}^{T} \Lambda_{k}\right) \widehat{x}_{k}^{T} \widehat{x}_{k} .
\end{aligned}
$$

For the eleventh term,

$$
\beta_{2}^{2} \sigma^{2} \widehat{x}_{k}^{T} \Lambda_{k} \widehat{x}_{k} \leq \beta_{2}^{2} \sigma^{2} \lambda_{\max }\left(\Lambda_{k}\right) \widehat{x}_{k}^{T} \widehat{x}_{k} .
$$

For the twelfth item

$$
\beta_{1}^{2} \sigma^{2} \Delta_{q}^{T} \Lambda_{k} \Delta_{q} \leq \beta_{1}^{2} \sigma^{2} \lambda_{\max }\left(\Lambda_{k}\right) \Delta_{q}^{T} \Delta_{q} .
$$

Therefore, 
12

Complexity

$$
\begin{aligned}
E\left\{V_{k+1}\left(e_{k+1}\right)\right\} \leq & \sum_{i=1}^{N} \varepsilon_{i}+\sum_{i=1}^{N}\left(1-\kappa_{i}\right) e_{i, k}^{T} \cdot P_{i, k}^{-1} \cdot e_{i, k}+\left[\left(\mu_{2}^{2} \beta_{1}^{2} \sigma^{2}+\mu_{2} \beta_{1} \sigma\right) \lambda_{\max }\left(\Lambda_{k}^{T} \Lambda_{k}\right)\right. \\
& \left.+\mu_{2}^{2} \beta_{1}^{2} \sigma^{2} \lambda_{\max }\left(\Lambda_{k}\right)+\mu_{2}^{2} \beta_{1} \beta_{2} \sigma^{2}\right] \lambda_{\max }\left(\mathscr{A}^{T} \mathscr{A}\right) \Delta \\
& +\left[\mu_{2} \beta_{1} \sigma+\mu_{2}^{2}\left(\beta_{1}^{2} \sigma^{2}+\beta_{1}^{2} \sigma^{2} \lambda_{\max }\left(\Lambda_{k}\right)+\beta_{1} \beta_{2} \sigma^{2}\right) \lambda_{\max }\left(\mathscr{L}^{T} \mathscr{L}\right)+\mu_{2} \beta_{2} \sigma\right] e_{k}^{T} e_{k} \\
& +\left[\left(\mu_{2} \beta_{2} \sigma+2 \mu_{2}^{2} \beta_{1} \beta_{2} \sigma^{2}\right) \lambda_{\max }\left(\Lambda_{k}^{T} \Lambda_{k}\right)+\mu_{2}^{2} \beta_{2}^{2} \sigma^{2} \lambda_{\max }\left(\Lambda_{k}\right)\right] \widehat{x}_{k}^{T} \widehat{x}_{k} \\
& +\mu_{2}^{2} \beta_{1}^{2} \sigma^{2} \lambda_{\max }\left(\Lambda_{k}\right) \Delta_{q}^{T} \Delta_{q} \\
\leq & {\left[1-m^{\prime}+\mu_{2} \bar{p}\left(\beta_{1}+\beta_{2}\right) \sigma+\mu_{2}^{2} \bar{p}\left(\beta_{1}^{2} \sigma^{2}+\beta_{1}^{2} \sigma^{2} \lambda_{\max }\left(\Lambda_{k}\right)+\beta_{1} \beta_{2} \sigma^{2}\right) \lambda_{\max }\left(\mathscr{L}^{T} \mathscr{L}\right)\right] e_{k}^{T} P^{-1} e_{k} } \\
& +\left[\left(\mu_{2}^{2} \beta_{1}^{2} \sigma^{2}+\mu_{2} \beta_{1} \sigma\right) \lambda_{\max }\left(\Lambda_{k}^{T} \Lambda_{k}\right)+\mu_{2}^{2} \beta_{1}^{2} \sigma^{2} \lambda_{\max }\left(\Lambda_{k}\right)+\mu_{2}^{2} \beta_{1} \beta_{2} \sigma^{2}\right] \lambda_{\max }\left(\mathscr{A}^{T} \mathscr{A}\right) \Delta \\
& +\left[\left(\mu_{2} \beta_{2} \sigma+2 \mu_{2}^{2} \beta_{1} \beta_{2} \sigma^{2}\right) \lambda_{\max }\left(\Lambda_{k}^{T} \Lambda_{k}\right)+\mu_{2}^{2} \beta_{2}^{2} \sigma^{2} \lambda_{\max }\left(\Lambda_{k}\right)\right] \Delta_{1} \\
& +\mu_{2}^{2} \beta_{1}^{2} \sigma^{2} \lambda_{\max }\left(\Lambda_{k}\right) \Delta_{2}+\sum_{i=1}^{N} \varepsilon_{i}
\end{aligned}
$$

where

$$
\begin{aligned}
\Delta_{1} & =x_{k}^{T} x_{k}, \\
\Delta_{2} & =\Delta_{q}^{T} \Delta_{q}, \\
m^{\prime} & =\min \left\{\kappa_{1}, \ldots, \kappa_{N}\right\} .
\end{aligned}
$$

$$
\lambda_{\max }\left(\Lambda_{k}^{T} \Lambda_{k}\right)=\lambda_{\max }\left(\Lambda_{k}^{2}\right)=\lambda_{\max }^{2}\left(\Lambda_{k}\right) \leq\left(\frac{1-m^{\prime}}{\underline{p}}\right)^{2}
$$

Therefore,

As shown in Lemma 3, we can obtain $\lambda_{\max }\left(\Lambda_{k}\right) \leq\left(\left(1-m^{\prime}\right) / \underline{p}\right)$.

$$
E\left\{V_{k+1}\left(e_{k+1}\right)\right\} \leq\left[1-m^{\prime}+\mu_{2} \bar{p}\left(\beta_{1}+\beta_{2}\right) \sigma+\mu_{2}^{2} \bar{p}\left(\beta_{1}^{2} \sigma^{2}+\beta_{1}^{2} \sigma^{2} \frac{1-m^{\prime}}{\underline{p}}+\beta_{1} \beta_{2} \sigma^{2}\right) \lambda_{\max }\left(\mathscr{L}^{T} \mathscr{L}\right)\right] V_{k}\left(e_{k}\right)+l^{\prime \prime}
$$

where

$$
\begin{aligned}
l^{\prime \prime}= & {\left[\left(\mu_{2}^{2} \beta_{1}^{2} \sigma^{2}+\mu_{2} \beta_{1} \sigma\right) \lambda_{\max }\left(\Lambda_{k}^{T} \Lambda_{k}\right)+\mu_{2}^{2} \beta_{1}^{2} \sigma^{2} \lambda_{\max }\left(\Lambda_{k}\right)+\mu_{2}^{2} \beta_{1} \beta_{2} \sigma^{2}\right] } \\
& \cdot \lambda_{\max }\left(\mathscr{A}^{T} \mathscr{A}\right) \Delta+\left[\left(\mu_{2} \beta_{2} \sigma+2 \mu_{2}^{2} \beta_{1} \beta_{2} \sigma^{2}\right) \lambda_{\max }\left(\Lambda_{k}^{T} \Lambda_{k}\right)+\mu_{2}^{2} \beta_{2}^{2} \sigma^{2} \lambda_{\max }\left(\Lambda_{k}\right)\right] \Delta_{1} \\
& +\mu_{2}^{2} \beta_{1}^{2} \sigma^{2} \lambda_{\max }\left(\Lambda_{k}\right) \Delta_{2}+\sum_{i=1}^{N} \varepsilon_{i} .
\end{aligned}
$$

Substituting (A.23) into Lemma 1,

$$
\alpha=m^{\prime}-\mu_{2} \bar{p}\left(\beta_{1}+\beta_{2}\right) \sigma-\mu_{2}^{2} \bar{p}\left(\frac{\beta_{1}^{2} \sigma^{2}+\beta_{1}^{2} \sigma^{2}\left(1-m^{\prime}\right)}{\underline{p}}+\beta_{1} \beta_{2} \sigma^{2}\right) \lambda_{\max }\left(\mathscr{L}^{T} \mathscr{L}\right) .
$$


Due to the conditions of Lemma 1, we know that

Then,

$$
\begin{aligned}
& 0<\alpha<1, \\
& \sum_{i=1}^{N} \varepsilon_{i}>0 .
\end{aligned}
$$

$$
0<m^{\prime}-\mu_{2} \bar{p}\left(\beta_{1}+\beta_{2}\right) \sigma-\mu_{2}^{2} \bar{p}\left(\frac{\beta_{1}^{2} \sigma^{2}+\beta_{1}^{2} \sigma^{2}\left(1-m^{\prime}\right)}{\underline{p}}+\beta_{1} \beta_{2} \sigma^{2}\right) \lambda_{\max }\left(\mathscr{L}^{T} \mathscr{L}\right)<1 .
$$

The condition $\sigma<\sigma^{*}$ makes the above quadratic inequality hold, where

$$
\sigma^{*}=\frac{-\bar{p}\left(\beta_{1}+\beta_{2}\right)+\left[\bar{p}^{2}\left(\beta_{1}+\beta_{2}\right)^{2}+\tau\right]}{2 \mu_{2}\left(\bar{p} \beta_{1}^{2}+\bar{p} \beta_{1}^{2}\left(1-m^{\prime}\right) / \underline{p}+\bar{p} \beta_{1} \beta_{2}\right) \lambda_{\max }\left(\mathscr{L}^{T} \mathscr{L}\right)},
$$

$$
\begin{array}{lr}
\text { where } \tau=4\left(\left(\bar{p} \beta_{1}^{2}+\bar{p} \beta_{1}^{2}\right.\right. & \left.\left.\left(1-m^{\prime}\right) / \underline{p}\right)+\bar{p} \beta_{1} \beta_{2}\right) \\
\left.\lambda_{\max }\left(\mathscr{L}^{T} \mathscr{L}\right) m^{\prime}\right]^{1 / 2} . & \square
\end{array}
$$

\section{Data Availability}

The figures .zip data used to support the findings of this study are available from the corresponding author upon request.

\section{Conflicts of Interest}

The authors declare that there are no conflicts of interest regarding the publication of this paper.

\section{Acknowledgments}

This work was supported by the Tianjin Natural Science Foundation (18JCYBJC42300) and Scientific Research Project of Tianjin Education Commission (2019KJ143).

\section{References}

[1] National Research Council, The Airliner Cabin Environment and the Health of Passengers and Crew, The National Academies Press, Washington, DC, USA, 2001.

[2] F. Li, J. Liu, J. Ren, X. Cao, and Y. Zhu, "Numerical investigation of airborne contaminant transport under different vortex structures in the aircraft cabin," International Journal of Heat and Mass Transfer, vol. 96, no. IV, pp. 287-295, 2016.

[3] S. C. Lee, C. S. Poon, X. D. Li, and F. Luk, "Indoor air quality investigation on commercial aircraft," Indoor Air, vol. 9, no. 3, pp. 180-187, 2010.

[4] G. Studor, "Fly-by-Wireless: a revolution in aerospace vehicle architecture for instrumentation and control," NASA technical report, Johnson Space Center, Houston, TX, USA, 2007.

[5] R. Priyanka, D. S. Kamal, C. Hankima, and M. B. Jean, "Wireless sensor networks: a survey on recent developments and developments and potential synergies," Journal Supercomputer, vol. 68, pp. 1-48, 2014.
[6] K. Sohraby, D. Minoli, and T. Znati, Wireless Sensor Networks: Technology, Protocols, and Applications, Wiley-Interscience, Hoboken. NJ, USA, 2007.

[7] W. Yang, X. Wang, and H. Shi, Optimal Consensus-Based Distributed Estimation with Intermittent communication, Taylor \& Francis, Inc., Milton Park, UK, 2011.

[8] R. Olfati-Saber, "Distributed Kalman filtering for sensor networks," in Proceedings of the IEEE Conference on Decision and Control, pp. 5492-5498, New Orleans, LA, USA, December 2007.

[9] R. Olfati-Saber, "Kalman-consensus filter: optimality, stability, and performance," in Proceedings of the IEEE Conference on Decision and Control, pp. 7036-7042, Shanghai, China, December 2009.

[10] D. H. Matthew and Z. H. Qu, "An optimal kalman-consensus filter for distributed implementation over a dynamic communication network," IEEE Access, pp. 66696-66706, 2021, inpress.

[11] M. Minyue Fu and L. Lihua Xie, "The sector bound approach to quantized feedback control," IEEE Transactions on Automatic Control, vol. 50, no. 11, pp. 1698-1711, 2005.

[12] A. Ribeiro and G. B. Giannakis, "Bandwidth-constrained distributed estimation for wireless sensor networks-part I: Gaussian case," IEEE Transactions on Signal Processing, vol. 54, no. 3, pp. 1131-1143, 2006

[13] A. Ribeiro and G. B. Giannakis, "Bandwidth-constrained distributed estimation for wireless sensor networks-part II: unknown probability density function," IEEE Transactions on Signal Processing, IEEE Press, vol. 54, no. 7, , pp. 2784-2796, 2006.

[14] T. Hayakawa, H. Ishii, and K. Tsumura, "Adaptive quantized control for linear uncertain discrete-time systems," Automatica, vol. 45, no. 3, pp. 692-700, 2009.

[15] H. Sun, N. Hovakimyan, and T. Basar, "L1 adaptive controller for systems with input quantization," in Proceedings of American Control Conference, IEEE, Baltimore, MD, USA, June 2010.

[16] T. Hayakawa, H. Ishii, and K. Tsumura, "Adaptive quantized control for nonlinear uncertain systems," in Proceedings of American Control Conference, June 2006.

[17] C. De Persis and F. Mazenc, "Stability of quantized time-delay nonlinear systems: a Lyapunov-Krasowskii-functional approach," Mathematics of Control, Signals, and Systems, vol. 21, no. 4, pp. 337-370, 2010.

[18] G. N. Nair, F. Fagnani, S. Zampieri et al., "Feedback control under data rate constraints: an overview," Proceedings of the IEEE, vol. 95, no. 1, pp. 108-137, 2007.

[19] D. F. Coutinho, M. Minyue Fu, and C. E. De Souza, "Input and output quantized feedback linear systems," IEEE Transactions on Automatic Control, vol. 55, no. 3, pp. 761-766, 2010. 
[20] R. Wang, X. Wang, H. Sun, Y. Huang, and Z. Chen, "Analysis of estimator and energy consumption with multiple faults over the distributed integrated WSN," International Journal of Modelling Identification and Control, vol. 32, no. 2, 2019.

[21] J. Liu, J. Xia, J. Cao et al., "Quantized state estimation for neural networks with cyber attacks and hybrid triggered communication scheme," Neurocomputing, vol. 291, pp. 3549, 2018.

[22] Z. G. Wu, Z. Xu, P. Shi et al., "Nonfragile state estimation of quantized complex networks with switching topologies," IEEE Transactions on Neural Networks and Learning Systems, vol. 29, no. 10, pp. 5111-5121, 2018.

[23] H. Ren, R. Lu, J. Xiong, and Y. Xu, "Optimal estimation for discrete-time linear system with communication constraints and measurement quantization," IEEE Transactions on Systems, Man, and Cybernetics: Systems, vol. 50, no. 5, pp. 1932-1942, 2020.

[24] M. Liu, L. Zhang, P. Shi, and Y. Zhao, "Fault estimation sliding-mode observer with digital communication constraints," IEEE Transactions on Automatic Control, vol. 63, no. 10, pp. 3434-3441, 2018.

[25] Z. Zhang, J. Li, and L. Liu, "Distributed state estimation and data fusion in wireless sensor networks using multi-level quantized innovation," Science China Information Sciences, vol. 59, no. 2, pp. 1-15, 2016.

[26] L. Hu, Z. Wang, and X. Liu, "Dynamic state estimation of power systems with quantization effects: a recursive filter approach," IEEE Transactions on Neural Networks and Learning Systems, vol. 27, no. 8, pp. 1604-1614, 2017.

[27] F. F. C. Rego, Y. Pu, A. Alessandretti et al., "Design of a distributed quantized luenberger filter for bounded noise," in Proceedings of American Control Conference, July 2016.

[28] P. Zoran, S. Milan, D. Milan, V. Nikola, D. Danijel, and M. Srdjan, "Floating point and fixed point 32-bits quantizers for quantization of weights of neural networks," in Proceedings of 12th International Symposium on Advanced Topics in Electrical Engineering (ATEE), Bucharest, Romania, March 2021.

[29] W. Li, Z. Xie, P. K. Wong, Y. Hu, G. Ge, and J. Zhao, "Eventtriggered asynchronous fuzzy filtering for vehicle sideslip angle estimation with data quantization and dropouts," IEEE Transactions on Fuzzy Systems, p. 1, 2021 inpress.

[30] H. Bian, Application of Modern Information Fusion Technology in Integrated Navigation System, National Defence Industrial Press, Beijing, China, 2010.

[31] R. Wang, Y. Li, H. Sun, and Z. Chen, "Analyses of integrated aircraft cabin contaminant monitoring network based on Kalman consensus filter," ISA Transactions, vol. 71, pp. 112120, 2017.

[32] K. Ranganathan, A Textbook of Graph Theory, Springer, Berlin, Germany, 2012.

[33] E. S. Biagioni and G. Sasaki, "Wireless sensor placement for reliable and efficient data collection," in Proceedings of IEEE 36th Annual Hawaii International Conference on System Sciences, 2003, p. 10, Big Island, HI, USA, January 2003.

[34] C. P. Njebla, "Topology and capacity planning for wireless heterogeneous networks in aircraft cabins," in Proceedings of IEEE International Symposium on Personal Indoor and Mobile Radio Communications, Helsinki, Finland, September 2006.

[35] J. Jin-Jun Xiao, S. Shuguang Cui, S. Zhi-Quan Luo, and A. J. Goldsmith, "Power scheduling of universal decentralized estimation in sensor networks," IEEE Transactions on Signal Processing, vol. 54, no. 2, pp. 413-422, 2006.
[36] K. Reif, S. Gunther, E. Yaz, and R. Unbehauen, "Stochastic stability of the discrete-time extended kalman filter," IEEE Transactions on Automatic Control, vol. 44, no. 4, pp. 714-728, 1999. 